Schields, R.L. (1976). A study of the validity of the educational administrative style diagnostic test. Unpublished doctoral dissertation, The American University.

Streiff, P. (1970). Some criteria for the evaluation of TESOL programs. TESOL Quarterly, 4, 365-370.

Wardhaugh, R. (1972). TESOL: Our common cause. TESOL Quarterly, 6, 291303.

Welch, D.F. (1976). A comparative study of the administrative styles of public and independent school principals and headmasters. Unpublished doctoral dissertation, The American University.

Wilcox, G.K. (1980, March). Suggested guidelines for the selection and/or preparation of ESOL program administrators. Paper presented at the 14 th Annual TESOL Convention, San Francisco.

Author's Address: Al Khazzan Street Residential Center, King Khalid Building, Apartment 1135, Riyadh, Saudi Arabia

\title{
The Effects of Modifying the Formality Level of ESL Composition Questions
}

\section{KEIKO HIROKAWA and JOHN SWALES \\ The University of Michigan}

We know of no investigations of the effect of topic on compositions written by ESL populations under closed-book, essay-examination conditions. In L1 contexts, several studies have been concerned with varying the amount and kind of information in the topic, or stimulus (e.g., Smith et al., 1985). In the most directly relevant of these studies, Brossell and Hoetker Ash (1984) investigated the effects of personal ("you") as opposed to neutral phrasing and of a question versus an imperative format. They found no significant results and concluded that their study "produced no evidence to support the contention that small changes in the wording of essay examinations of otherwise similar construction affect writers or the holistic ratings given their essays" (p. 425).

The study reported here examined the extent to which the short and general topics used in the Composition section of the Michigan English Language Assessment Battery (MELAB) (English Language Institute, 1984) generate rhetorically and linguistically unambitious answers that fail to indicate fully the communicative abilities of candidates. Since this investigation was part of a series of studies aimed at developing a new test specifically designed for the evaluation of nonnative-speaking (NNS) graduate and transfer students, it also examined the extent to which more ambitious and more academically appropriate writing could be elicited by simply increasing the level of formality of the topic. An example of simple and academic topic variants is given below. 
Simple: Would you prefer to be part of a large family or a small one?

Academic: Family size tends to vary according to a number of factors, such as culture, religion, mortality rate, and level of economic development. What are the advantages and disadvantages of small "nuclear" families as opposed to larger extended family units? State your personal preference for one of these family types and explain the reasons behind that preference.

The subjects were 32 NNSs enrolling at the University of Michigan in Fall 1985. Each subject wrote two compositions under test conditions. In the first condition, the subjects wrote on academic topics as part of their English proficiency reevaluation (time limit 45 minutes). Two weeks later, the same subjects-23 of whom were enrolled in an academic writing course and 9 of whom had been exempted from ESL writing classes - took a version of the standard MELAB Composition, responding to simple topics which were different in content area from the topics in the first condition (time limit 30 minutes).

Following standard MELAB scoring procedures, all compositions were scored by two experienced raters. Mean MELAB scores were 77.4 for the simple-topic condition and 78.5 for the academic-topic, with scores ranging from 67 to 93 .

The two sets of performances were analyzed through paired $t$ tests, and statistically significant differences at the .01 level were found for several variables: Simple-topic compositions (a) were longer than the academic topic compositions, as measured by both words written per 30 minutes and sentences written per 30 minutes; (b) contained more subordination (per standardized length); (c) exhibited greater use of the first-person, singular pronoun; and $(\mathrm{d})$ contained more morphological errors.

The following variables were found to be significant at the .05 level: Simple-topic compositions exhibited a lower proportion of Graeco-Latin vocabulary (per standardized length), as measured by procedures developed by Corson (1982), and had higher proportions of total errors and of syntactic errors. Nonsignificant variables included sentence length and frequency of logical connectors, second-person pronouns, and the passive voice.

Interviews with 5 of the subjects revealed their strong general preference for the more academic and elaborate questions because, as one interviewee put it, "it is easier to understand what they want us to write." Interestingly, the academic-topic sessions did not give rise to as many preliminary queries and requests for clarification as is customary in standard MELAB test sessions.

A general comparison of performance in the two formality conditions reveals relatively few and relatively small differences ascribable to the 
experimental variable. Some of these differences are predictable enough, such as the increased use of first-person pronouns when responding to simple and general topics and the correlation between increased speed of writing and increased error rate. On the other hand, the enhanced utilization of Graeco-Latin lexis in the academic-topic condition is potentially interesting, and the amount of subordination is not easy to reconcile with general expectations.

There was evidence of at least two subgroups: one apparently capable of some variation in its rhetorical and stylistic response to the level of formality of the stimulus (most strikingly revealed in the contrasting opening paragraphs) and one apparently unable, unwilling, or unaware of the need to match a response to the level of formality of the stimulus. While it is not clear whether this capacity for variation correlates with writing ability, as measured by MELAB scores, there was certainly no relationship between students' self-perception (as manifested in the five interviews) and their actual writing performance.

The verdict of this study would seem to be the Scottish one of "not proven," especially as the research design did not permit equal writing time in the two conditions. On the other hand, it should be noted that in both conditions, advice to candidates about assessment criteria remained the same: "You will be graded on how well you communicate your ideas, not on your ideas themselves." The effect of changing this advice in various ways is currently being investigated, for it would seem easier to communicate well ideas of lesser cognitive complexity. ${ }^{1}$

\section{REFERENCES}

Brossell, A., \& Hoetker Ash, B. (1984). An experiment with the wording of essay questions. College Composition and Communication, 35, 423-425.

Corson, D.J. (1982). The Graeco-Latin (G-L) instrument: A new measure of semantic complexity in oral and written English. Language and Speech, 25, 1-10.

English Language Institute, Testing and Certification Division. (1984). Michigan English language assessment battery. Ann Arbor: University of Michigan.

Smith, W. L., Hull, G. A., Land, R. E., Moore, M. T., Ball, C., Dunham, D. E., Hickey, L. S., \& Ruzich, C. W. (1985). Some effects of varying the structure of a topic on college students' writing. Written Communication, 2: 73-89.

Authors' Address: English Language Institute, North University Building 2001, The University of Michigan, Ann Arbor, MI 48109

1This study was funded by the Testing and Certification Division of the English Language Institute.

BRIEF REPORTS AND SUMMARIES 